\title{
Surgical and Functional Outcomes of Sigmoid Vaginoplasty Among Patients With Variants Of Disorders of Sex Development
}

\author{
Nowier A, Esmat M, Hamza RT \\ Departments of Urology (NA, EM) and Pediatrics (HRT), Faculty of Medicine, Ain Shams University, \\ Cairo, Egypt
}

\section{ABSTRACT}

Purpose: To assess the use of sigmoid colon in vaginal reconstruction of some patients with disorders of sex development.

Materials and methods: The study included 31 patients with disorders of sex development of various causes. All were reared as females. Female gender was decided for all cases after complete medical assessment. All patients underwent sigmoid vaginoplasty. Assessment of surgical and functional outcomes was carried out in a follow up period of up to 6 years.

Results: The preoperative diagnoses included mullerian aplasia (16 cases), androgen insensitivity syndrome (12 cases) and previous failed vaginoplasty (3 cases). Associated surgical procedures were gonadectomy in 5 cases and gonadectomy combined with clitoroplasty and vulvoplasty in 7 cases. No intra-operative or early postoperative complications occurred. A cosmetic neovagina with adequate size was achieved in all cases. Long term follow up showed introital stenosis in 4 cases $(12.9 \%)$. Two of them responded to vaginal dilatation. The third one needed $\mathrm{y}-\mathrm{v}$ plasty while the fourth one presented by acute abdomen secondary to ruptured vagina and was submitted to urgent laparotomy. Mucosal prolapse occurred in 1 case $(3.2 \%)$. Reoperation rate was 9.6\%. Sexual satisfaction was achieved among 9 sexually active cases. The subjective satisfaction score of the surgical outcome was 8.03.

Conclusions: For patients with disorders of sex development of various etiologies, sigmoid vaginoplasty is the preferred technique for vaginal replacement. It is a safe technique that provides the patient with a cosmetic neovagina of adequate caliber and a satisfactory functional outcome.
ARTICLE INFO

Key words:

Colon; Sigmoid;

Sex Reassignment Surgery;

Genitalia; Abnormalities;

Disorders of Sex Development

Int Braz J Urol. 2012; 38: 380-88

Submitted for publication:

May 30, 2011

Accepted after revision:

October 28, 2011

\section{INTRODUCTION}

The reconstruction of a neovagina is indicated for cases of congenital absence of vagina, genetic sexual ambiguity and vaginal loss resulting from gynecologic cancer or post traumatic injury $(1,2)$.
The ideal surgical technique for vaginoplasty is the one that can provide the patient with a vaginal vault of sufficient size, adequate introitus and an acceptable cosmetic external appearance (3).

Several surgical techniques were described for vaginal replacement. Baldwin was 
the first one to describe the use of intestinal segment for vaginoplasty in 1904 (4). Depending on this principle, Wallace was able to use the sigmoid colon successfully in 1911 (5). Later on, this procedure was discontinued due to its high morbidity rate (6). In the last few decades, the use of bowel segment for vaginoplasty was reviewed including laparoscopic approach after the reported high complication rate of other methods of vaginal reconstruction (7). In this series, we evaluate the use of sigmoid colon for vaginal replacement among patients with disorders of sex development (DSD).

\section{MATERIALS AND METHODS}

The current study was conducted from February 2003 to May 2009 at Ain Shams University Hospitals, Cairo, Egypt. It included 31 patients submitted to sigmoid vaginoplasty. Their preoperative diagnosis was mullerian aplasia (16 cases), androgen insensitivity syndrome (12 cases) (5 cases were complete and 7 cases had incomplete androgen insensitivity) and 3 cases with previous failed vaginoplasty (by skin graft) done elsewhere for treatment of vaginal aplasia.

All patients were submitted to careful history taking and physical examination. Twenty one patients seeked medical treatment due to primary amenorrhea while 7 patients (with incomplete androgen insensitivity) due to ambiguous genitalia. The remaining 3 patients with failed previous vaginoplasty had a severely stenotic vagina

All patients (except secondary cases) underwent karyotyping, pelvi-abdominal ultrasonography, pelvic CT or MRI, endocrinal and psychiatric assessment. An informed written consent was obtained from all patients or their legal guardians. The study was approved by the ethical committee of Ain Shams University Hospitals, Cairo, Egypt. All patients underwent mechanical and antibiotic bowel preparation 48 hours prior to surgery.

\section{Surgical procedure}

Under general or epidural anesthesia and in an extended lithotomy position, creation of a large and enough space between rectum and bladder at the site of perineal dimple was performed using progressively larger Hegar dilators. Next, a midline subumbilical incision was performed to select, mobilizate and isolate a segment of $12-15 \mathrm{~cm}$ of the distal sigmoid and its vascular bed (Figures 1A and B). The remained colon was reanastomosed. The cul-de-sac was opened over a Hegar dilator pushed through the perineal route. The isolated colonic segment was closed by 2 layers at its proximal end that was fixed to the sacral promontory. The distal end was pulled thoroughout the abdomino-perineal pouch to the perineum where it was sutured to vulvar mucosa (Figure-2A). The peritoneum was closed above the transposed bowel and the neovagina was molded with a vaginal stent (loose vaseline vaginal pack) for 48 hours (Figure-2B).

Associated procedures included gonadectomy in all cases of androgen insensitivity syndrome and clitoroplasty with vulvoplasty in the 7 cases with incomplete androgen insensitivity syndrome.

Postoperatively, all patients underwent vaginal dilatation program in the form of self dilatation and irrigation daily for 10 weeks then weekly thereafter until the patient became sexually active.

Follow-up was done from 6 months to 6 years. Physical examination to assess vaginal length and width, cosmetic appearance of the neovagina and occurrence of any complications were performed. The grade of satisfaction of the surgical outcome was estimated by a subjective satisfaction score (range from $0=$ very disappointed to $10=$ satisfied) (8). Personal interviews (done by another urologist of our department) were carried out for assessment of the functional outcome among the sexually active patients. Vaginogram was performed in all patients at 6 months postoperatively. Vaginoscopy was performed annually in all patients for early detection of malignancy.

\section{RESULTS}

The current study included 31 patients aged 14-28 years (mean age $18.55 \pm 3.63$ years). 
Figure 1 - (A): Isolation of $12-15 \mathrm{~cm}$ of sigmoid colon, (B): The bowel segment has been positioned to be anastomosed to vulvar mucosa.

A

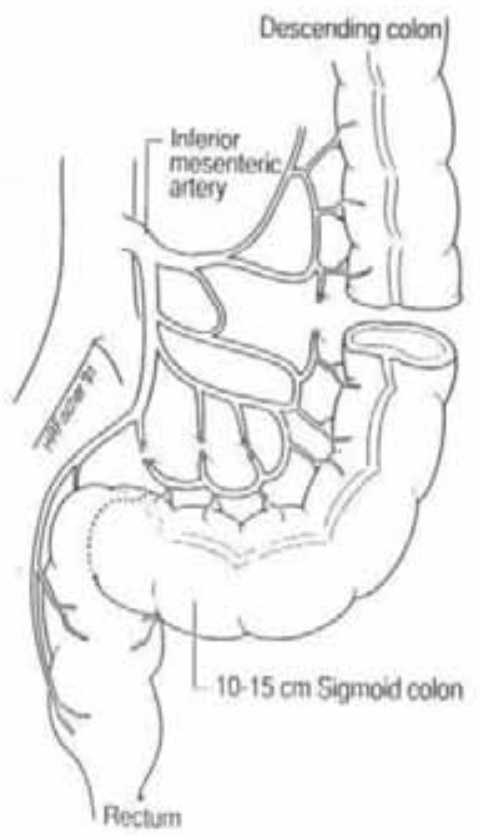

B

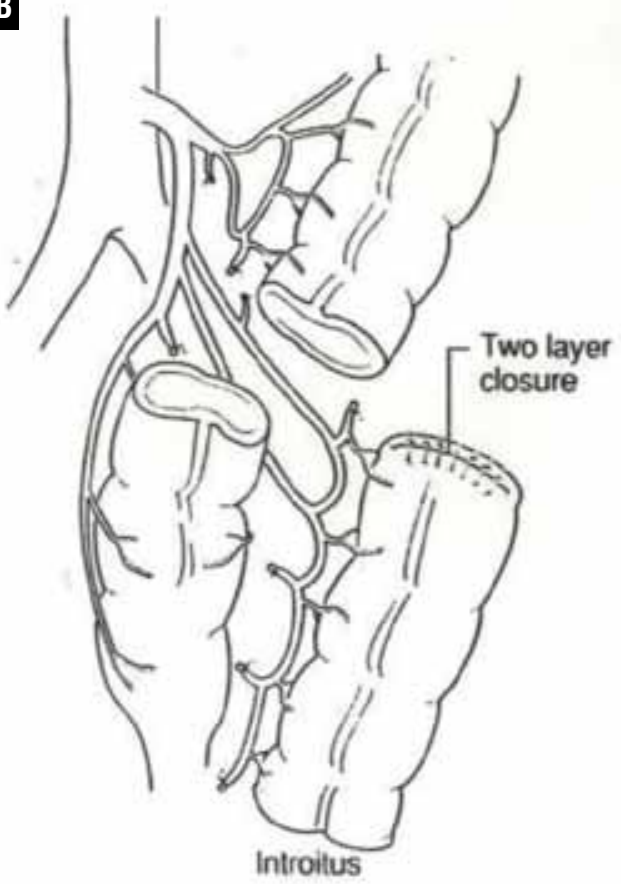

Figure 2 A and B - (A) Colocolostomy has been completed and the distal end of the colonic segment has been anastomosed to the opened rudimentary vaginal pit, (B): The peritoneum was closed above the transposed bowel and the neovagina was loosely packed with Vaseline gauze.

A

ANTESTINAL VAGINOPLASTY

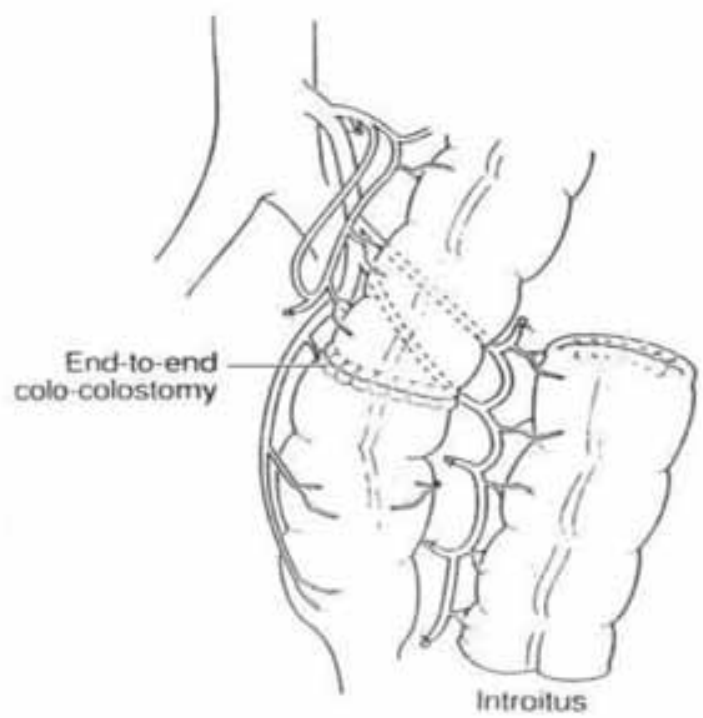

B

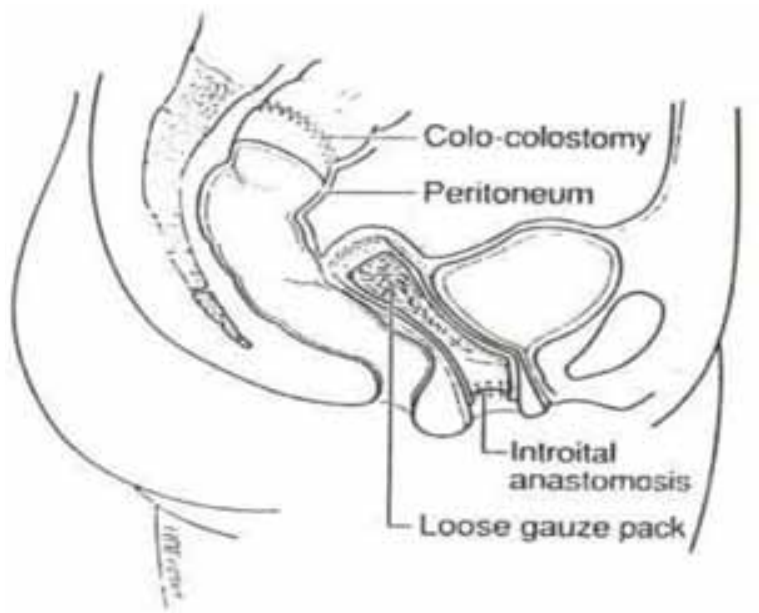


The preoperative evaluation revealed 16 cases with mullerian aplasia (Figure-3A). Their karyotype was 46XX. Hormonal profile regarding follicular stimulating hormone, luteinizing hormone and estradiol were normal according to age and sex matched reference ranges (9). On ultrasonography, a rudimentary or absent uterus was found. Another 12 cases were diagnosed as androgen insensitivity syndrome. All were reared as females. Their karyotype was 46XY. Inguinal gonads were felt on physical examination. Their serum testosterone level was elevated in comparison to normal age- and sex- matched reference ranges (10). Seven cases of them seeked medical attention due to ambiguous genitalia and were diagnosed as incomplete androgen insensitivity (Figure-3B). The other 5 cases who complained of primary amenorrhea were diagnosed as complete androgen insensitivity. No sexual ambiguity was found on their physical examination. The remaining 3 cases had a history of previous failed vaginoplasty. Their physical examination revealed a contractured stenotic vagina. Two of them had an ugly perineal scar (Figure-3C) secondary to previous failed vaginoplasty done by a full thickness groin skin graft.

All cases underwent sigmoid vaginoplasty. The mean operative time was 142 minutes (range 110-205 minutes). No intra-operative complications occurred and the postoperative period was uneventful. The patients were discharged from the hospital within 6 - 8 days.

During follow up, the neovagina was found to have an excellent cosmetic appearance (Figure-4). The mean vaginal length was $13.0 \mathrm{~cm}$ (range $10.5-15 \mathrm{~cm}$ ). Vaginograms showed that all patients achieved a neovagina of adequate length and caliber (Figure-5).

Excessive vaginal discharge was found in all patients during the first 2 months postoperatively, however, it ceased markedly within the next $1-3$ months. Three patients complained of malodor of the vaginal discharge that was managed conservatively by vaginal irrigation.

Figure 3 A to C - Some preoperative diagnoses of studied groups. (A): a case of mullerian aplasia, (B): a case of incomplete androgen insensitivity syndrome and (C): a recurrent case with stenosed vagina and scarred perineum.
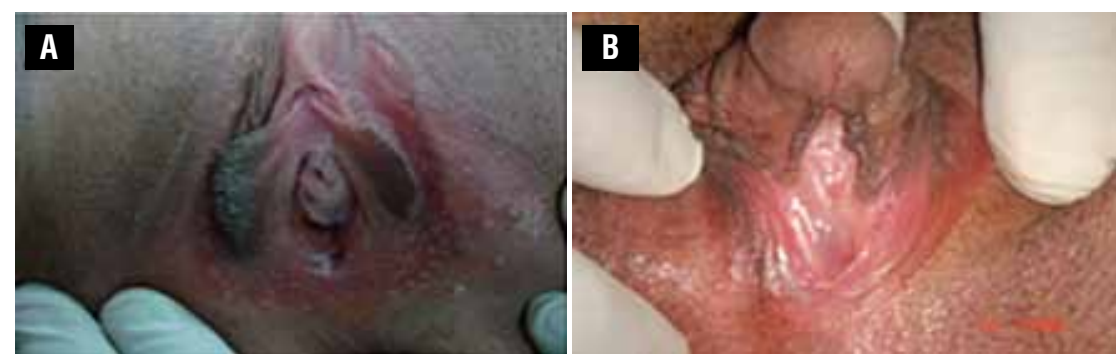

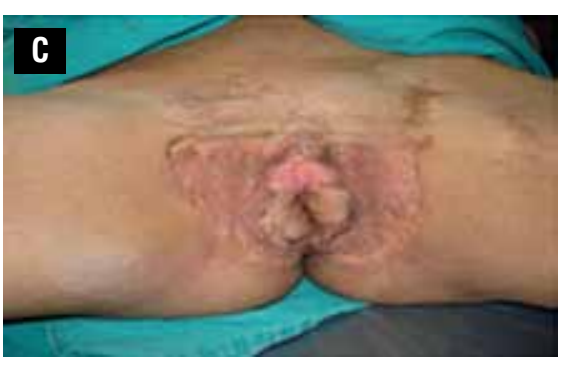

Figure 4 - A postoperative case of sigmoid vaginoplasty.

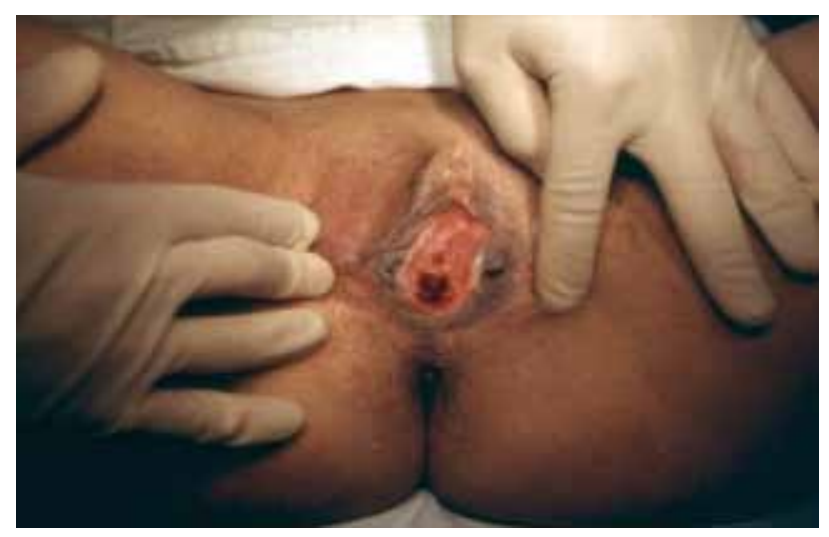

Figure 5 - Postoperative vaginogram after sigmoid vaginoplasty.

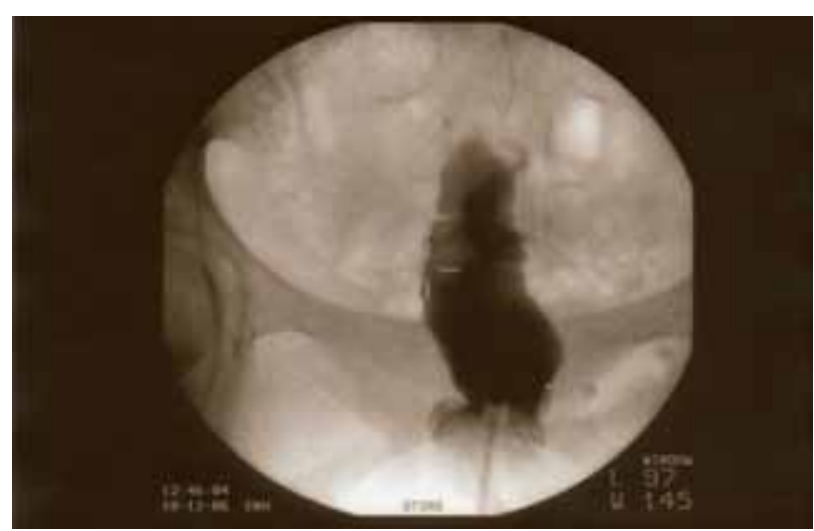


Long term follow up revealed that 4 patients $(12.9 \%)$ had vaginal introital stenosis; 2 of those cases responded to dilation while one case needed $\mathrm{y}-\mathrm{v}$ plasty. The remaining patient had an acute $\mathrm{ab}-$ domen secondary to vaginal rupture that needed urgent laparotomy. Mucosal prolapse occurred in 1 patient that required surgical resection of the prolapsed tissue. The rate of surgical intervention for complications was 9.6\%.

During follow up, most patients were satisfied with the surgical outcome. Mean satisfaction subjective score was 8.02 (range 6-9). Nine cases were sexually active and reported a satisfactory sexual life. Two cases with previous failed vaginoplasty regained their sexual activity 10 weeks postoperatively. Mild dyspareunia was experienced by 3 cases initially during the first few intercourses that progressively disappeared later on. None of the cases developed colitis or adenocarcinoma.

\section{DISCUSSION}

Proper diagnosis is essential for the management of patients with DSD. All patients in the current series were reared as females. After clinical, endocrinal and psychiatric assessments, female gender was confirmed and vaginal reconstruction was indicated for all of them.

Many methods of vaginal reconstruction were reported. The non operative technique which is known as Frank procedure can be used when a vaginal dimple or pouch is present and involves a progressive mechanical dilation using graduated hard dilators to create a progressive invagination of the vaginal dimple (11). Other surgical techniques reported depended on the creation of a perineal cleavage covered by a selected tissue. They include McIndoe procedure which involves insertion of a mold covered with split thickness skin graft taken from the buttocks into the created neovaginal space followed by postoperative vaginal dilation (12). Others used full thickness skin graft from the buttocks or skin flap based on labia majora (13), peritoneum from the Douglas pouch (14), amnion (15), oxidized regenerated cellulose fabric (16) and muscle flaps e.g. pudendale- thigh flap (17). The high percentage of vaginal stenosis, inadequate vaginal length, vaginal dryness and dyspareunia were reported as drawbacks of these techniques. Moreover, these modalities require long term vaginal dilatation and stenting by a vaginal mold at night which affects the patient's psychological condition passively; these modalities must be avoided in pediatric age groups.

Intestinal segment either sigmoid, ileum or ileocecal segment can be used for vaginal replacement. Concerning the use of ileocecal segment for vaginoplasty, it is known that it can lead to metabolic disturbance and must be avoided in pediatric patients. Anatomically, the sigmoid colon is closest to the perineum and can be pulled easily with its vascular bed to the perineum and its diameter is sufficient for vaginoplasty without reconfiguration. On the other hand, the ileal mucosa was found to be more fragile and may lead to mild bleeding after intercourse. In addition, the mucus production by the ileum is more excessive and less lubricating than that of the sigmoid (18). Moreover, vaginal stenosis was found to be more frequent with ileal than sigmoid vaginoplasty (19).

In our series, no intra-operative or intestinal complications occurred. Increased morbidity with sigmoid vaginoplasty due to occurrence of intestinal leakage and intestinal obstruction was reported by another study in 3.2\% of patients (18). Intestinal complications are rarely reported with sigmoid vaginoplasty and we suggest that preoperative bowel preparation, previous experience with bowel surgery and meticulous suturing are essential prerequisites to avoid such complications.

Four cases (12.9\%) in this study developed stenosis at the mucocutaneous junction. Two of them were managed by progressive vaginal dilatation under anesthesia followed by regular weekly self dilatation. Another case was found to have a tight vaginal stenosis that required surgical intervention in the form of Y-V plasty. The fourth case presented in the emergency room with acute abdomen secondary to rupture vagina due to retained secretions (mucocele). Exploration revealed intraperitoneal rupture of the neovagina. Excision of the devitalized tissue, repair of the vagina, drainage of the peritoneum and dilatation of the stenosed vaginal orifice were done. Thus, regular follow up and vaginal dilatation are essential for prevention of such a hazardous complication. Variable incidence of introital 
stenosis was reported with sigmoid vaginoplasty: (8.1\% (18) and 19.3\% (20)). The liability for anastomotic stricture can be decreased by selection of a bowel segment of adequate blood supply and length that can be mobilized and pulled easily to the perineum without tension, creation of a large enough space between bladder and rectum and generous anastomosis at the hymenal region. The need for regular vaginal dilatation after sigmoid vaginoplasty was advised by some authors (21) while others did not recommend it (22). When we analyzed our data, we found that all cases with vaginal stenosis were sexually inactive and reluctant to perform self vaginal dilatation .Therefore, we propose that regular postoperative dilatation of the anastomotic site till full sexual activity (once/week at least) is preferred to decrease the incidence of this complication.

In our series, one case of mucosal prolapse (3.2\%) was observed and managed by surgical excision of redundant tissue. Another study reported mucosal prolapse in $7.6 \%$ of patients (21). Full thickness vaginal prolapse that required retroperitoneal fixation was reported by others (23). This complication rarely happens and can be avoided by selection of appropriate bowel segment length and proper fixation to the sacral promontory thus decreasing the incidence of this complication.

Excess mucosal discharge with malodour was reported by some authors in 8.3\% of patients after rectosigmoid vaginoplasty (24) while others reported no mucous problem $(25,26)$. In our series, this was not a problem as this secretion decreased markedly within 2-5 months postoperatively. Thereafter, the patients experienced just a moist vagina with no complaint. The malodor of vaginal secretions that was experienced by 3 patients was managed easily by regular vaginal irrigation 2-3 times weekly.

On vaginal examination of our patients, we found that all patients had an excellent cosmetic appearance of the neovagina with an adequate vaginal orifice. Vaginal calibration and vaginograms revealed an adequate size of the neovagina in all patients. Patients were satisfied with their surgical outcome. The estimated mean subjective satisfaction score was 8.03. Moreover, a cosmetic neovagina of adequate size was also obtained among the 3 recurrent cases with previous failed vaginoplasty using skin graft so we suggest the use of sigmoid vaginoplasty in secondary cases although the limited number of this patient's category in our series. Other studies also reported good cosmetic and functional results of sigmoid vaginoplasty $(22,27)$ whereas other authors reported periumbilical pain during intercourse in 5.6\% of patients and considered this pain a disadvantage of this procedure (24). With assessment of the functional outcome among the 9 sexually active patients in our series, none of them complained of this pain whereas good results were obtained as all patients experienced intercourses that were satisfactory for them and for their partners. No significant dyspareunia or bleeding during intercourse was reported. None of the patients complained of vaginal dryness or need for external lubricants but instead they experienced a moist vagina due to the colonic mucosal secretion.

None of our patients developed ulcerative colitis which was reported in another study (28). Also, diversion colitis did not occur. This is a rare disorder of unknown etiology that may occur after isolation of an intestinal segment from the fecal stream (29). The risk of adenocarcinoma of the intestinal neovagina was reported and found to be rare and lower than the risk of cancer after Abbe-McIndoe procedure (30). None of our patients developed adenocarcinoma during the follow up period. Annual follow up is advisable for early detection of malignancy.

\section{CONCLUSIONS}

Our study revealed that sigmoid vaginoplasty can provide the patient with a self lubricating, esthetically pleasing neovagina of adequate size. It has a low complication rate and a low incidence of interoital stenosis with no need for daily vaginal dilatation or vaginal stenting by vaginal molds. Moreover, the reported functional outcome is excellent.

\section{CONFLICT OF INTEREST}

None declared. 


\section{REFERENCES}

1. ACOG Committee on Adolescent Health Care. ACOG Committee Opinion. Number 274, July 2002. Nonsurgical diagnosis and management of vaginal agenesis. Obstet Gynecol. 2002; 100: 213-6.

2. Evans TN, Poland ML, Boving RL: Vaginal malformations. Am J Obstet Gynecol. 1981; 141: 910-20.

3. Powell DM, Newman KD, Randolph J: A proposed classification of vaginal anomalies and their surgical correction. $J$ Pediatr Surg. 1995; 30: 271-5; discussion 275-6.

4. Baldwin JF. XIV: The Formation of an Artificial Vagina by Intestinal Trransplantation. Ann Surg. 1904; 40: 398-403.

5. Goligher JC: The use of pedicled transplants of sigmoid or other parts of the intestinal tract for vaginal construction. Ann R Coll Surg Engl. 1983; 65: 353-5.

6. Fall FH: A simple method for making an artificial vagina. Am J Obstet Gynecol 1940; 40: 906-17.

7. Li B, Wang J, Wu JX, Wang LY: Clinical analysis of vaginoplasty with sigmoid colon by laparoscopic surgery. Zhonghua Fu Chan Ke Za Zhi. 2009; 44: 673-5.

8. Wewers ME, Lowe NK: A critical review of visual analogue scales in the measurement of clinical phenomena. Res Nurs Health. 1990; 13: 227-36.

9. Elmlinger MW, Kühnel W, Ranke MB: Reference ranges for serum concentrations of lutropin (LH), follitropin (FSH), estradiol (E2), prolactin, progesterone, sex hormone-binding globulin (SHBG), dehydroepiandrosterone sulfate (DHEAS) cortisol and ferritin in neonates, children and young adults. Clin Chem Lab Med. 2002; 40: 1151-60.

10. Sato $Y$, Tanda $H$, Kato $S$, Onishi $S$, Nakajima $H$, Nanbu $A$, et al.: Serum testosterone levels using the radioimmunoassay method in healthy Japanese male volunteers. Reprod Med Biol 2006; 5: 37-41.

11. Frank RT: The formation of an artificial vagina without operation. Am J Obstet Gynec. 1938; 35: 1053-5.

12. McIndoe A: The treatment of congenital absence and obliterative conditions of the vagina. Br J Plast Surg. 1950; 2: 254-67.

13. Sadove RC, Horton CE: Utilizing full-thickness skin grafts for vaginal reconstruction. Clin Plast Surg. 1988; 15: 443-8.

14. Sheth NP, Chainani MS, Sheth SN: Vaginoplasty from peritoneal tube of Douglas' pouch for congenital vaginal agenesis. Eur J Pediatr Surg. 2003; 13: 213-4.

15. Ashworth MF, Morton KE, Dewhurst J, Lilford RJ, Bates RG: Vaginoplasty using amnion. Obstet Gynecol. 1986; 67: 443-6.

16. Jackson ND, Rosenblatt PL: Use of Interceed Absorbable Adhesion Barrier for vaginoplasty. Obstet Gynecol. 1994; 84: 1048-50.

17. Joseph VT: Pudendal-thigh flap vaginoplasty in the reconstruction of genital anomalies. J Pediatr Surg. 1997; 32: 62-5.
18. Imparato E, Alfei A, Aspesi G, Meus AL, Spinillo A: Longterm results of sigmoid vaginoplasty in a consecutive series of 62 patients. Int Urogynecol J Pelvic Floor Dysfunct. 2007; 18: 1465-9.

19. Hensle TW, Dean GE: Vaginal replacement in children. J Urol. 1992; 148:677-9.

20. Hensle TW, Reiley EA: Vaginal replacement in children and young adults. J Urol. 1998; 159: 1035-8.

21. Khen-Dunlop N, Lortat-Jacob S, Thibaud E, Clément-Ziza M, Lyonnet S, Nihoul-Fekete $C$, et al.: Rokitansky syndrome: clinical experience and results of sigmoid vaginoplasty in 23 young girls. J Urol. 2007; 177: 1107-11.

22. Moudouni S, Koutani A, Attya Al, Hachimi M, Lakrissa A: The use of isolated sigmoid colon segment for vaginal replacement in young adults. Int Urol Nephrol. 2004; 36: 567-71.

23. Freundt I, Toolenaar TA, Jeekel H, Drogendijk AC, Huikeshoven FJ: Prolapse of the sigmoid neovagina: report of three cases. Obstet Gynecol. 1994; 83(Pt 2): 876-9.

24. Kwun Kim S, Hoon Park J, Cheol Lee K, Min Park J, Tae Kim J, Chan Kim M: Long-term results in patients after rectosigmoid vaginoplasty. Plast Reconstr Surg. 2003; 112: 143-51.

25. Ekinci S, Karnak I, Ciftci AO, Senocak ME, Tanyel FC, Büyükpamukçu N: Sigmoid colon vaginoplasty in children. Eur J Pediatr Surg. 2006; 16: 182-7.

26. Kapoor R, Sharma DK, Singh KJ, Suri A, Singh P, Chaudhary $H$, et al.: Sigmoid vaginoplasty: long-term results. Urology. 2006; 67: 1212-5.

27. Piro C, Asensio M, Martín JA, Giné C, Ormaetxea E, Chicaiza $\mathrm{E}$ : Sigmoid colon vaginoplasty: experience with five cases. Cir Pediatr. 2006; 19: 19-22.

28. Froese DP, Haggitt RC, Friend WG: Ulcerative colitis in the autotransplanted neovagina. Gastroenterology. 1991; 100: 1749-52.

29. Toolenaar TA, Freundt I, Huikeshoven FJ, Drogendijk AC, Jeekel $\mathrm{H}$, Chadha-Ajwani S: The occurrence of diversion colitis in patients with a sigmoid neovagina. Hum Pathol. 1993; 24: 846-9.

30. Hiroi H, Yasugi T, Matsumoto K, Fujii T, Watanabe T, Yoshikawa $\mathrm{H}$, et al.: Mucinous adenocarcinoma arising in a neovagina using the sigmoid colon thirty years after operation: a case report. J Surg Oncol. 2001; 77: 61-4.

Correspondence address: Dr. Mohamed Esmat Departments of 1 Urology, Faculty of Medicine, Ain Shams University, Cairo, Egypt Fax: + 202 2690-4430 E-mail: m_esmat_2000@yahoo.com 


\section{EDITORIAL COMMENT}

The authors report on a retrospective study of high number of patients who underwent sigmoid vaginoplasty. They show, as do many others authors, that this surgery is effective. The cosmetic result is good and most often patients are able to have a normal sexual life. Despite there being no new information in this paper, the data presented add to the literature. However, only nine patients were sexually active and the time of follow is still not long enough to catch some complications. Outcomes that should be evaluated with this procedure include: cosmetics, vaginal discharge of mucus, adequate canal vaginal diameter, absence of dispareunia and orgasm.

Cosmetics results are usually good with this procedure, because the bowel-vaginal anastomosis is generally concealed. The authors performed a midline abdominal incision. However, abdominal scars have also to be counted in the

\section{EDITORIAL COMMENT}

Congenital genitourinary and intersex disorders such as Mayer-Rokitansky-Kuster-Hauser syndrome, androgen insensitivity, congenital adrenal hyperplasia, and gonadal dysgenesis result in vaginal agenesis or atresia (1). Attainment long-term functional and cosmetic outcomes with low morbidity makes reconstructive procedures a challenge for surgeons. The recommended nonsurgical Frank method by the American College of Obstetrics and Gynecology is not applicable in all cases because of the requirement for long period of vaginal dilations and subsequent surgical treatment in most of treated patients $(2,3)$.

The technique used in this research by Nowier and colleagues is based on the use of cosmetic consideration. This procedure can be easily carried out with a low transverse abdominal incision or even by laparoscopy, regardless of the age of the patients. Vaginal mucus is commonly transitory and the vaginal diameter generally accomodates penetration without discomfort. Four patients had vaginal introital stenosis. The authors did not mention the age of the patients during surgery or whether or not they were sexually active. Also, the time of diagnosis of stenosis is not reported. When performing this procedure in sexually active patients, the vaginal penetration acts as a natural dilation and helps reduce this kind of complication. The authors reported that 9 cases were sexually active and that all had a satisfactory sexual life. The term satisfactory is vague, and information regarding number of sexual relations per month and the presence of orgasm should be stated in future trials.

Dr. Ubirajara Barroso Jr. Universidade Federal da Bahia - UFBA, Brazil Email:ubarroso@uol.com.br

bowel graft to create a neovagina and was first described by Baldwin in 1904 (4). The authors reported their experience on the treatment of 31 patients aged 14-28 years with variants of disorders of sex development using sigmoid vaginoplasty with acceptable follow up period. The paper is worth reading as it reports functional and sexual data of this procedure. The authors address a useful technical point to fix the proximal end of the neovagina to the sacral promontory in order to prevent prolapse.

Sigmoid colon is the most common segment used when there is isolated vaginal absence. This technique is more complex than other reconstructive vaginoplasty surgeries; however, 
improvements in technique and postoperative care permitted the use of sigmoid graft as firstline surgical therapy (3). The optimal timing of surgery remains a source of controversy. Many surgeons who favor the use of bowel for neovagina reconstruction do not believe that vaginoplasty should be deferred until the patient reaches adulthood (5).

One of the advantages of this procedure is the possibility of coitus shortly after surgery; in comparison to the technique of passive dilation which required an average delay of 12 months (6). Carrard et al. evaluated sexuality results of

\section{REFERENCES}

1. O'Connor JL, DeMarco RT, Pope JC 4th, Adams MC, Brock JW 3rd: Bowel vaginoplasty in children: a retrospective review. J Pediatr Surg. 2004; 39: 1205-8.

2. ACOG Committee on Adolescent Health Care: ACOG Committee Opinion No. 355: Vaginal agenesis: diagnosis, management, and routine care. Obstet Gynecol. 2006; 108: 1605-9.

3. Carrard C, Chevret-Measson M, Lunel A, Raudrant D: Sexuality after sigmoid vaginoplasty in patients with MayerRokitansky-Küster-Hauser syndrome. Fertil Steril. 2012; 97 691-6. the procedure with the use of two standardized questionnaires: the Female Sexual Function Index (FSFI) and the revised Female Sexual Distress Scale (FSDS-R). They concluded that this technique provides a nearly normal sexual function for patients. Also they showed that psychologic distress related to sexuality persists in most cases and suggested a multidisciplinary support for them (3).

Herein we can appreciate an important study improving our knowledge regarding the outcome of this technique. Further studies should investigate the long term follow up with more number of cases.
4. Baldwin JF: XIV. The Formation of an Artificial Vagina by Intestinal Trransplantation. Ann Surg. 1904; 40: 398-403.

5. Hendren WH, Atala A: Use of bowel for vaginal reconstruction. J Urol. 1994; 152: 752-5; discussion 756-7.

6. Roberts $\mathrm{CP}$, Haber MJ, Rock JA: Vaginal creation for müllerian agenesis. Am J Obstet Gynecol. 2001; 185: 1349-52; discussion 1352-3.

Dr. Aida Moeini

Department of Obstetrics and Gynecology, Shohada Tajrish Hospital, Shaheed Beheshti Medical Sciences University Tehran, Iran

Dr. Mohammad Mohsen Mazloomfard Department of Urology, Shohada Tajrish Hospital, Shaheed Beheshti Medical Sciences University, Tehran, Iran E-mail: mazloomfard@yahoo.com 